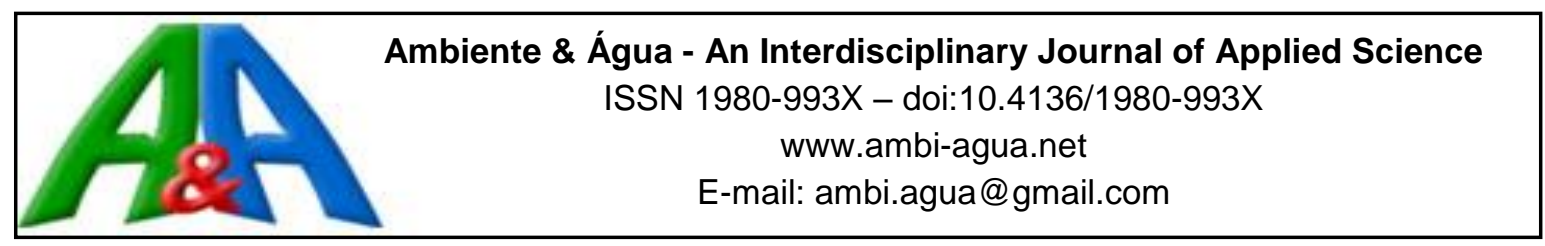

\title{
Remoção de cistos de Giardia spp. e oocistos de Cryptosporidium spp. na Estação de Tratamento de Esgoto Garcia, no município de Blumenau, SC, Brasil
}

\author{
doi:10.4136/ambi-agua.2028
}

Received: 18 Oct. 2016; Accepted: 30 Aug. 2017

\section{Mauro Giovanni Miglioli ${ }^{*}$; Jessica Geremias Zuanazzi²; Joel Dias da Silva ${ }^{3}$; Regina Maura Bueno Franco ${ }^{4}$; Juliane Araújo Greinert-Goulart ${ }^{2}$}

\author{
${ }^{1}$ Fundação Universidade Regional de Blumenau (FURB), Blumenau, SC, Brasil \\ Programa de Pós-Graduação em Engenharia Ambiental (PPGEA). E-mail: mauro.miglioli@ hotmail.com \\ ${ }^{2}$ Fundação Universidade Regional de Blumenau (FURB), Blumenau, SC, Brasil \\ Departamento de Ciências Naturais (DCN). E-mail: jzuanazzi.comercial@gmail.com, julianeag@gmail.com \\ ${ }^{3}$ Fundação Universidade Regional de Blumenau (FURB), Blumenau, SC, Brasil \\ Departamento de Engenharia de Produção e Design. E-mail: dias_joel@ hotmail.com \\ ${ }^{4}$ Universidade Estadual de Campinas (UNICAMP), Campinas, SP, Brasil \\ Departamento de Biologia Animal. E-mail: mfranco@unicamp.br \\ *Autor correspondente
}

\section{RESUMO}

Giardia spp. e Cryptosporidium spp. são agentes etiológicos responsáveis por uma série de epidemias de gastroenterites ocorridas, principalmente, após o consumo de água contaminada. Os cistos e oocistos destes protozoários são resistentes às variações ambientais, bem como a maioria dos processos físicos, químicos e microbiológicos utilizados nas estações de tratamento de água e esgoto. Deste modo, este estudo teve como objetivo detectar e avaliar a remoção de cistos de Giardia spp. e oocistos de Cryptosporidium spp. no sistema de tratamento combinado anaeróbio + aeróbio da ETE Garcia em Blumenau, SC. Para efetuar a detecção de cistos e oocistos, as amostras de efluentes e lodos provenientes da ETE foram concentradas através de filtração em membranas de ésteres de celulose e centrifugação, seguida por reação de imunofluorescência direta (RID) utilizando o Kit diagnóstico - Merifluor ${ }^{\circledR}$. Para a obtenção dos parâmetros físicos, químicos e microbiológicos, as análises seguiram os padrões preconizados em APHA (2012). Concentrações elevadas de cistos de Giardia spp. (máx. 900.000 cistos $\mathrm{L}^{-1}$ ) foram detectadas em $83,3 \%$ dos pontos analisados. Oocistos de Cryptosporidium spp. também foram detectados em elevadas concentrações (máx. 300.000 oocistos $\mathrm{L}^{-1}$ ) em $66,6 \%$ dos pontos analisados. Não foram detectados cistos e oocistos nas amostras do efluente tratado, deste modo o sistema combinado da ETE Garcia apresentou uma eficiência $>99,9 \%$ para a remoção das formas resistentes destes patógenos, contribuindo para a redução da contaminação ambiental por protozoários patogênicos presentes no esgoto doméstico do município de Blumenau, SC, Brasil.

Palavras-chave: eficiência de remoção, esgoto doméstico, protozoários de veiculação hídrica. 


\title{
Removal of Cryptosporidium spp. oocysts and Giardia spp. cysts at a Waste Water Treatment Plant Garcia, in Blumenau, SC, Brazil
}

\begin{abstract}
Giardia spp. and Cryptosporidium spp. are responsible for a number of outbreaks of gastroenteritis, particularly associated with the consumption of contaminated water. The cysts and oocysts of these protozoa are resistant to environmental variations, as well as to most of the physical, chemical and microbiological processes used in water treatment and sewage plants. This study therefore aimed to detect and evaluate the removal of Giardia spp. and Cryptosporidium spp. in a combined system anaerobic / aerobic Waste Water Treatment Plant (WWTP) located in the city of Blumenau, SC, Brazil. For the study of cysts and oocysts, samples of effluents and sludge from the WWTP were concentrated by filtration using a membrane of cellulose esters and by centrifugation, followed by direct immuno-fluorescence assay (RID) using the Merifluor ${ }^{\circledR}$ diagnostic kit. The analysis followed the standards published in APHA (2012) to obtain the physical, chemical and microbiological parameters. High concentrations of Giardia spp. (Max. $900,000 \mathrm{~L}^{-1}$ cysts) were detected in $83.3 \%$ of the analyzed points. Oocysts Cryptosporium spp. were detected at high concentrations (max. 300,000 oocysts $\mathrm{L}^{-1}$ ) in $66.6 \%$ of the analyzed points. Neither cysts nor oocysts were detected in treated effluent samples. Thus, the combined system of the Garcia WWTP showed a 99.9\% efficiency rate for the removal of resistant forms of pathogens, contributing to the reduction of environmental contamination by these pathogens present in the domestic sewage of Blumenau, SC.
\end{abstract}

Keywords: domestic sewage, removal efficiency, waterborne protozoa.

\section{INTRODUÇÃO}

A água pode ser responsável pela veiculação de uma variedade de doenças a população humana, quando contaminada e utilizada para consumo, irrigação, pesca ou recreação, resultando nas chamadas doenças de veiculação hídrica (Cheng et al., 2009).

Giardia spp. e Cryptosporidium spp. são conhecidos como parasitos cosmopolitas (Fregonesi et al., 2012) e agentes causais da giardiose e criptosporidiose respectivamente, com infecções associadas à indivíduos imunocomprometidos, como também aos imunocompetentes (Chappell et al., 2015). Estão entre as principais causas de doenças diarreicas em seres humanos em todo o mundo e são considerados para a Saúde Pública, os mais importantes protozoários patogênicos veiculados pela água, devido à elevada resistência à maioria dos processos convencionais para o tratamento de água potável, resistência ao cloro e a alta infectividade (Putignani e Menichella, 2010; WHO, 2011; Fregonesi et al., 2012). Esses protozoários potencialmente patogênicos também são resistentes à maioria dos processos empregados para o tratamento de esgoto doméstico (Nasser et al., 2012; Nasser, 2016). As formas infectantes destes patógenos estão presentes nas fezes dos hospedeiros infectados, que compõem o esgoto bruto que chega às ETE (Estação de Tratamento de Esgoto), tornando o esgoto doméstico, uma das principais fontes de contaminação dos mananciais (Nasser et al., 2012; Nasser, 2016).

Deste modo, Giardia spp. e Cryptosporidium spp. foram agentes patogênicos de 524 surtos de doenças de veiculação hídrica, que ocorreram em todo o mundo no período compreendido de 1984 a 2010 (Karanis et al., 2007; Baldursson e Karanis, 2011).

Outro cenário associado a esses protozoários além da veiculação hídrica é a transmissão por alimentos, deste modo a Organização das Nações Unidas para Alimentação e a Agricultura (Food and Agriculture Organization - FAO) em parceria com a Organização Mundial da Saúde 
(World Health Organization - WHO) classificou Cryptosporidium spp. com a $5^{\circ}$ posição do ranking das mais importantes zoonoses globais de origem alimentar e Giardia duodenalis na $11^{\circ}$ posição (FAO e WHO, 2014).

Devido à relevância destas enfermidades para a saúde pública, no ano de 2004, a criptosporidiose e a giardiose foram incluídas na lista "Iniciativa das Doenças Negligenciadas", da Organização Mundial de Saúde (OMS) e Organização Pan-Americana de Saúde (OPAS), pois, restringem o desenvolvimento e a melhoria socioeconômica de vários países (Savioli et al., 2006).

O Fundo das Nações Unidas para a Infância, em parceria com a Organização Mundial da Saúde, aponta as doenças diarreicas como a segunda maior causa de mortes em crianças menores de 5 anos de idade em todo o mundo. Estima-se que 1,5 milhões de crianças são levadas a óbito a cada ano vítimas destas doenças, sendo $88 \%$ destes óbitos associados à sistemas inadequados de saneamento básico, sendo que $99 \%$ destes obtidos ocorrem em países em desenvolvimento (UNICEF e WHO, 2009).

Atualmente, mais de 748 milhões de pessoas em todo o mundo não possuem serviços para a disponibilidade de água potável, e aproximadamente 2,5 bilhões pessoas não possuem serviços de coleta e tratamento do esgoto sanitário (WHO, 2014).

No Brasil, 38,7\% dos esgotos gerados recebem algum tipo de tratamento (SNIS, 2014). No Estado de Santa Catarina, apenas 21,35\% (Trata Brasil, 2013). Já no município de Blumenau, os serviços de coleta e tratamento de esgotos sanitários atendem cerca de $33 \%$ da população (Blumenau, 2016).

No município de Blumenau, Grott et al. (2016) relataram a presença de Giardia spp. e Cryptosporidium spp. em águas superficiais brutas destinadas ao abastecimento público, e Andrade et al. (2008) como parasito infectante da população humana infantil. E no município de Indaial, Volotão et al. (2008) identificaram Giardia duodenalis subtipo A1 (infectante para humanos) como parasito infectante de animais silvestres, porém, nenhum trabalho foi realizado na região visando detectar a presença destes protozoários em amostras de efluentes domésticos brutos, tratados ou em subprodutos deste tratamento, como os lodos biológicos, evidenciando a necessidade de pesquisas que investiguem possíveis ambientes de disseminação destes protozoários.

Deste modo, o presente trabalho teve como objetivo detectar e avaliar a eficiência de remoção de cistos de Giardia spp. e oocistos de Cryptosporidium spp. no sistema combinado anaeróbio + aeróbio da ETE Garcia; em Blumenau/SC.

\section{MATERIAL E MÉTODOS}

\subsection{Caracterização da ETE Garcia}

A ETE Garcia está localizada no Bairro Garcia, inserida no vale do Ribeirão de mesmo nome e a Sudeste do município de Blumenau, SC (Figura 1).

A estação contempla, com coleta e tratamento de esgoto, aproximadamente 36.846 habitantes dos bairros Garcia, Ponta Aguda, Centro, Vorstadt e Valparaíso. Utiliza sistema combinado para o tratamento de efluentes, constituída de uma estação elevatória, sistema de gradeamento mecanizado (AGUAGARD ${ }^{\circledR}$ ), desarenador (AIRLIFTH ${ }^{\circledR}$ ), calha do tipo Parshall para a medição de vazão e pH do esgoto bruto, reator UASB (Upflow Anaerobic Sludge Blanket - reator anaeróbio de fluxo ascendente) com sistema de captação e queima de gases, reator MBBR (Moving Bed Biofilm Reactor - reator biológico de leito móvel), decantador secundário, tanque de contato (desinfecção), sistema de recirculação e centrifugação de lodo (Figura 2). 


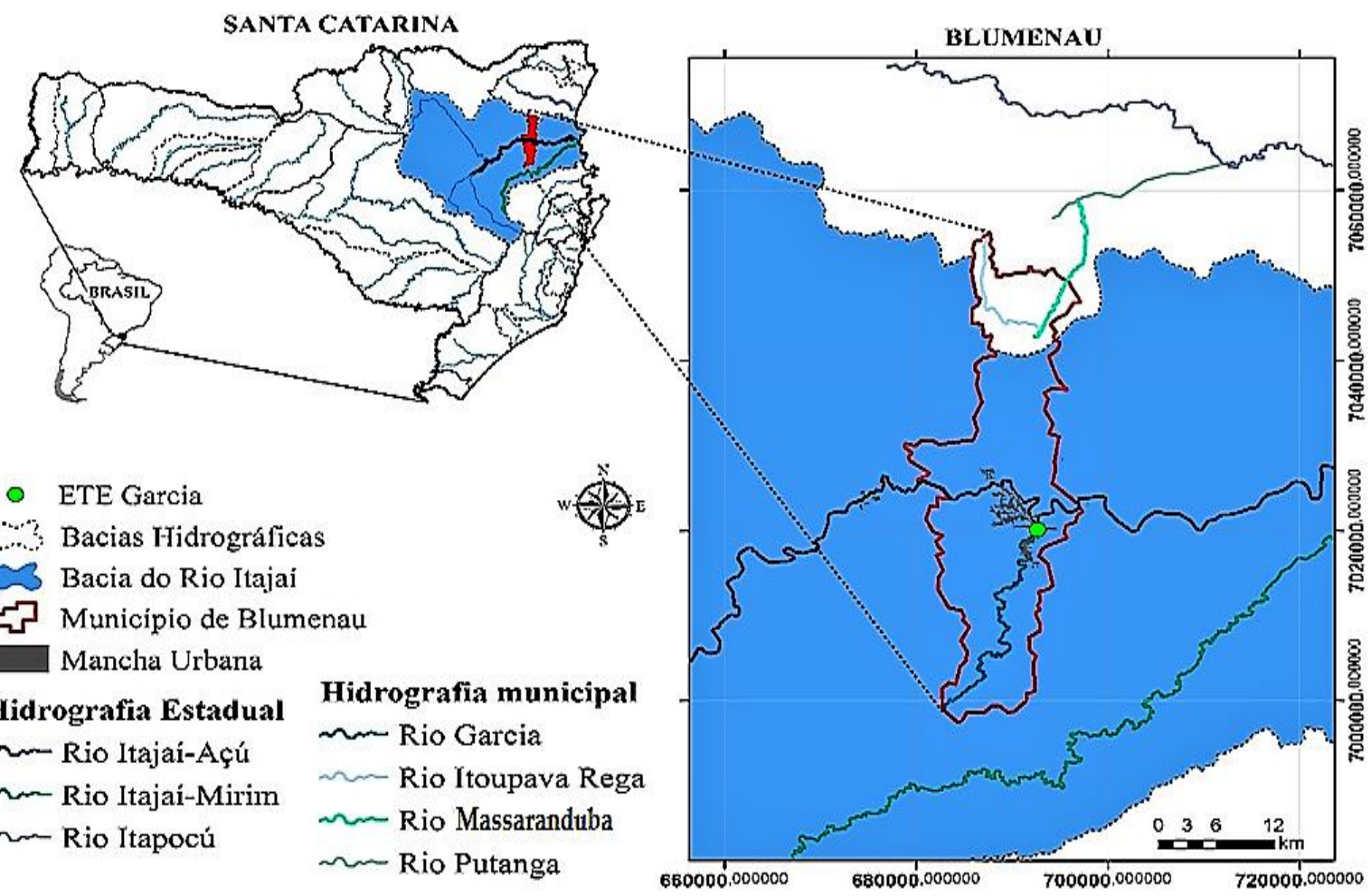

Figura 1. Localização da ETE Garcia, em Blumenau/SC, Brasil.

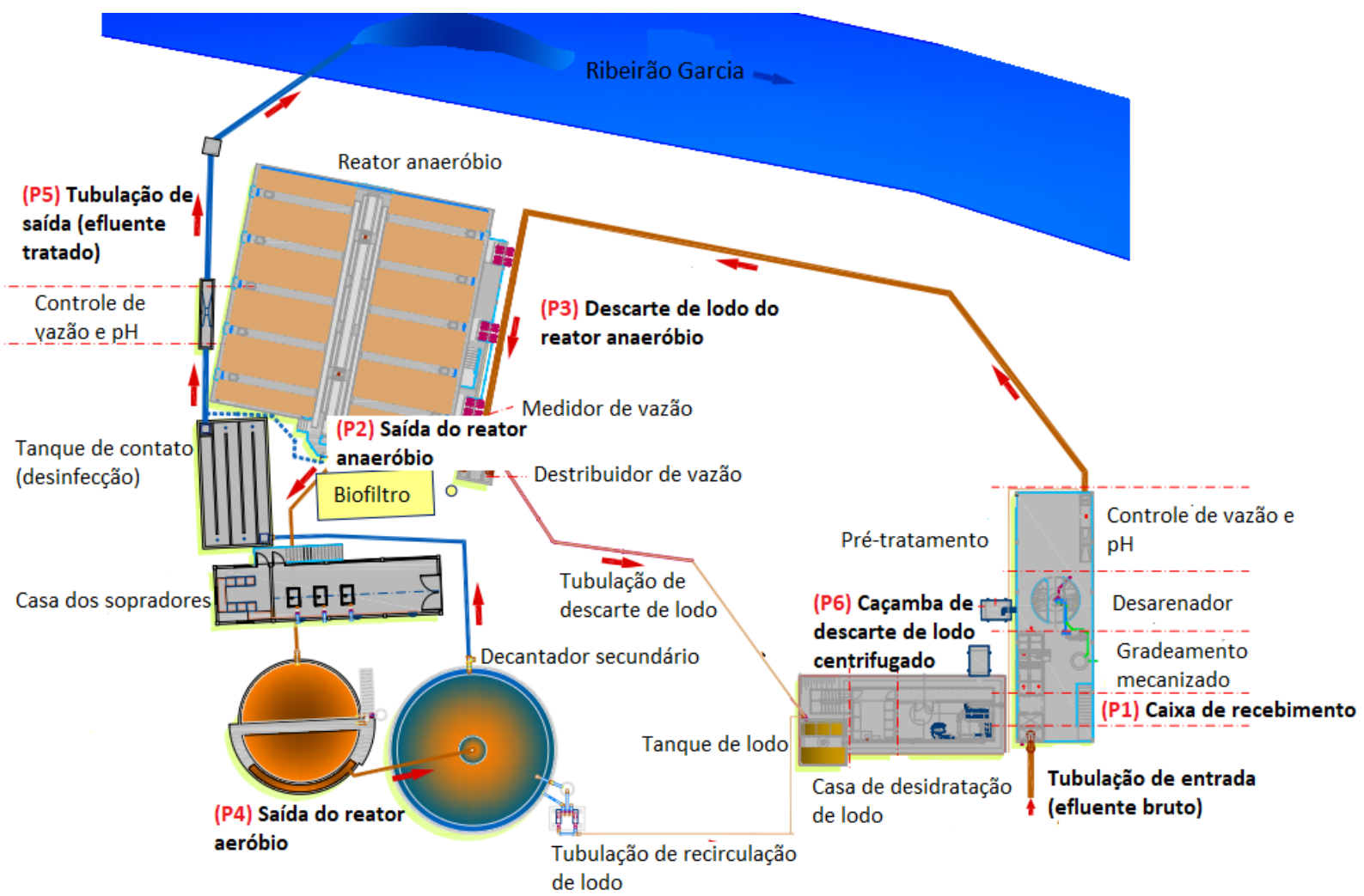

Figura 2. Planta baixa do sistema de tratamento de esgoto do Bairro Garcia.

Fonte: adaptado de Odebrecht (2016). 
A estação foi projetada com capacidade de tratamento de $88 \mathrm{~L} \cdot \mathrm{s}^{-1}$, mas, atualmente opera

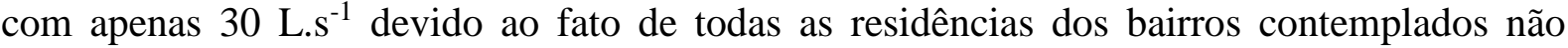
estarem interligadas a rede coletora.

\subsection{Amostragem}

As análises parasitológicas, físicas, químicas e microbiológicas foram realizadas mensalmente durante um período de 12 meses tendo início em fevereiro de 2015 e término em janeiro de 2016.

As amostras de efluentes e lodos foram armazenadas em galões plásticos estéreis, previamente higienizados e lavados com solução de eluição contendo Tween 80 a 0,01\% (1:3). Estas amostras foram coletadas de acordo com o tempo de detenção hidráulica (TDH) da ETE Garcia que varia em função da vazão que entra na mesma, sendo a vazão média mensal de 30 L.s- ${ }^{1}$, e TDH de aproximadamente $34 \mathrm{~h}$.

Deste modo o primeiro ponto de coleta foi o efluente bruto (P1), o segundo ponto de coleta foi na saída do efluente UASB, (P2) o terceiro no lodo UASB (P3), o quarto ponto de coleta foi na saída do efluente MBBR (P4), o quinto ponto de coleta foi na saída da estação no efluente tratado (P5) que é lançado no ribeirão Garcia, e o sexto ponto de coleta foi o lodo condicionado da estação (P6) representados na Figura 2.

\subsection{Detecção de cistos de Giardia spp. e oocistos Cryptosporidium spp.}

As análises realizadas nesta pesquisa foram conduzidas no Laboratório de Parasitologia do Departamento de Ciências Naturais e no Laboratório de Engenharia de Biomassa (LEBIO), do Programa de Pós-Graduação em Engenharia Ambiental (PPGEA) da Universidade Regional de Blumenau (FURB), SC, Brasil.

Os cistos de Giardia spp. e oocistos de Cryptosporidium spp. eventualmente presentes nas amostras de efluente bruto, lodo do reator UASB, efluente do reator MBBR e lodo condicionado foram concentradas de acordo com protocolo modificado de Robertson et al. (2000) e adaptado por Cantusio Neto et al. (2006).

Em tubos de $15 \mathrm{~mL}$ foram adicionados $5 \mathrm{~mL}$ das amostras previamente filtradas em peneiras de $1 \mathrm{~mm}^{2}$, adicionados $10 \mathrm{~mL}$ de Solução de Eluição contendo Tween 80 a 0,1\% (1:3) e homogeneizado por 2 minutos com auxílio de Vórtex (KASVI ${ }^{\circledR}$ - K45-2820).

Posteriormente estas amostras foram centrifugadas a $1.500 \mathrm{x} g$ durante 15 minutos, com o auxílio da centrifuga (HERMLE ${ }^{\circledR}$ - Z326K), o sobrenadante foi aspirado com o auxílio de uma bomba a vácuo (TECNAL ${ }^{\circledR}$ - TE-0581). O sedimento precipitado foi armazenado em microtubos de $2 \mathrm{~mL}$, com a adição antibióticos (gentamicina, penicilina e anfotericina $\mathrm{B}$ ) e armazenados a $4^{\circ} \mathrm{C}$

As amostras de efluente tratado e efluente do reator UASB foram concentradas conforme protocolo modificado de Franco et al. (2001). As amostras foram filtradas em membranas de ésteres de celulose com $47 \mathrm{~mm}$ de diâmetro e porosidade de $3 \mu \mathrm{m}$ (Milipore ${ }^{\circledR}$ ), através de um sistema de filtração com bomba a vácuo (fluxo 4 L.min ${ }^{-1}$ ) e porta filtro (Gelman ${ }^{\circledR}$ ).

Após a filtração as membranas foram cuidadosamente retiradas e transferidas para placas plásticas estéreis para realização da recuperação de cistos de Giardia spp. e de oocistos Cryptosporidium spp. As superfícies das membranas foram raspadas por 20 minutos com o auxílio de alças plásticas alternadamente de lavagens manuais com solução de eluição. $\mathrm{O}$ material resultante das lavagens foi concentrado através de dupla centrifugação a $1.050 \times \mathrm{x}$ durante 15 minutos por meio da centrifuga. O sobrenadante foi aspirado e descartado e o sedimento lavado com solução de eluição e centrifugado novamente. O sobrenadante foi aspirado e descartado e o sedimento precipitado foi armazenado em microtubos de $2 \mathrm{~mL}$ com a adição de antibióticos e armazenados a $4^{\circ} \mathrm{C}$.

A pesquisa de cistos de Giardia spp. e oocistos Cryptosporidium spp. foi realizada por 
meio da reação de imunofluorescência direta (RID) conforme as instruções do fabricante do Kit diagnóstico (Merifluor ${ }^{\circledR}$ - Meridian Bioscience, Cincinnatti, Ohio). A visualização da RID foi realizada empregando o microscópio de epifluorescência (Olympus ${ }^{\circledR}$ - CH30) com filtro de excitação de 450 a $490 \mathrm{~nm}$ e filtro de barreira de $520 \mathrm{~nm}$.

Após a enumeração dos cistos e oocistos presentes nas amostras, foi realizado o cálculo da estimativa de número médio de cistos e oocistos $\mathrm{L}^{-1}$, de acordo com a Equação 1 descrita por Cantusio Neto e Franco (2004).

$$
\mathrm{X}=\frac{\mathrm{N}}{\mathrm{K}} \times \frac{\mathrm{S}}{\mathrm{A}}
$$

em que:

$\mathrm{X}$ : concentração de oocistos ou cistos $\mathrm{L}^{-1}$;

$\mathrm{N}$ : número de oocistos ou cistos visualizados na lâmina;

$\mathrm{K}$ : volume de sedimento analisado $(\mu \mathrm{L})$;

S: volume total do sedimento obtido $(\mu \mathrm{L})$;

A: volume filtrado da amostra (L).

O cálculo da remoção de cistos e oocistos foi realizado utilizando-se as concentrações de cistos e oocistos detectados no esgoto bruto e no efluente final da ETE (Equação 2).

$\%$ remoção $=C P 5.100 / C P 1$

em que:

CP1: concentração de cistos ou oocistos no esgoto bruto (P1)

CP5: concentração de cistos ou oocistos no eflente tratado (P5)

Durante todo o período das análises parasitologicas, entre o processamento das amostras, foram realizados experimentos utilizando água reagente como amostra, seguindo os mesmos procedimentos e materiais utilizados nas amostras naturalmente investigadas. Neste experimento não foram detectados cistos ou oocistos, demonstrando que não houve contaminação dos equipamentos e materiais utilizados.

\subsection{Parâmetros físicos, químicos e microbiológicos}

Os seguintes parâmetros físicos, químicos e microbiológicos foram avaliados na entrada (efluente bruto) e na saída (efluente tratado) da ETE Garcia: Demanda Bioquímica de Oxigênio - DBO5 (fotometria); Demanda Química de oxigênio - DQO (fotometria); Turbidez (nefelometria); Temperatura (termometria), Sólidos sedimentáveis - SD60 (gravimetria); Sólidos suspensos totais - STT (gravimetria); Sólidos totais - ST (gravimetria); pH (sonda); coliformes totais e coliformes termotolerantes (membrana filtrante).

As metodologias na obtenção dos parâmetros físicos e químicos e microbiológicos seguiram os padrões publicados por APHA (2012). Estes parâmetros físicos, químicos e microbiológicos foram correlacionados com a concentração de cisto de Giardia spp. e de oocisto de Cryptosporidium spp.

\subsection{Análise dos resultados}

Para a série de dados, aplicou-se o teste de Shapiro Wilk a fim de testar a normalidade. Os resultados da pesquisa de cistos de Giardia spp. e oocistos de Cryptosporidium spp. nas diversas amostras da ETE Garcia foram analisados utilizando estatística descritiva: média aritmética, 
desvio padrão e valores máximos e mínimos, utilizando o software Excel 2016.

Para a análise da variação sazonal da concentração de cistos de Giardia spp. e oocistos de Cryptosporidium spp. nas amostras analisadas foi aplicado o teste ANOVA de fator único.

Para a análise da existência de correlação entre os resultados da pesquisa de cistos Giardia spp. e oocistos de Cryptosporidium spp. e os dados físicos, químicos e microbiológicos das amostras analisadas foi aplicado o teste não-paramétrico de coeficiente de correlação de postos de Spearman $(r)$ utilizado o software STATISTICA 10.0 (StatSoft). Em relação à concentração de cisto de Giardia spp. e oocistos de Cryptosporidium spp., para a análise de correlação, foi realizada uma previa transformação logarítmica dos dados, utilizando $\log (\mathrm{y}+1)$ e posteriormente aplicado o Teste $\mathrm{t}$ de Student $(p)$ para verificar a significância do valor de $(r)$. Somente valores de $\mathrm{p} \leq 0,05$ foram considerados significantes.

\section{RESULTADOS E DISCUSSÃO}

\subsection{Parâmetros físicos, químicos e microbiológicos}

Durante a realização desta pesquisa o efluente bruto (entrada) e o efluente tratado (saída) da ETE Garcia apresentaram as seguintes concentrações médias referentes aos parâmetros físicos, químicos e microbiológicos (Tabela 1).

Tabela 1. Parâmetros físico-químicos e microbiológicos do efluente bruto, efluente tratado e eficiência de remoção da ETE Garcia, município de Blumenau, SC.

\begin{tabular}{|c|c|c|c|c|c|c|}
\hline \multirow{2}{*}{$\begin{array}{l}\text { Amostras/ } \\
\text { Variáveis }\end{array}$} & \multirow{2}{*}{$\mathrm{n}$} & \multicolumn{2}{|c|}{ Efluente bruto (entrada) } & \multicolumn{2}{|c|}{ Efluente tratado (saída) } & \multirow{2}{*}{$\begin{array}{l}\text { Eficiência } \\
\text { de remoção } \\
(\%)\end{array}$} \\
\hline & & Amplitude & Média \pm DP & Amplitude & Média \pm DP & \\
\hline DBO5 & 12 & $(7-352)$ & $(196 \pm 105)$ & $(4-34)$ & $(14 \pm 10)$ & 91,0 \\
\hline DQO & 12 & $(432-1835)$ & $(780 \pm 385)$ & $(2-186)$ & $(71 \pm 40)$ & 91,0 \\
\hline $\mathrm{pH}$ & 12 & $(6,5-7,2)$ & $(6,9 \pm 0,19)$ & $(6,9-7,5)$ & $(7,2 \pm 0,19)$ & - \\
\hline SD60 & 12 & $(0,6-39)$ & $(7,01 \pm 10,4)$ & $(0,1-0,1)$ & $(0,1 \pm 0)$ & 99,0 \\
\hline STT & 12 & $(146-280)$ & $(204 \pm 37)$ & $(3-60)$ & $(17 \pm 15)$ & 91,0 \\
\hline ST & 12 & $(565-985)$ & $(715 \pm 104)$ & $(256-410)$ & $(335 \pm 42)$ & 52,0 \\
\hline Temperatura & 12 & $(24-29)$ & $(26 \pm 1,7)$ & $(21-33)$ & $(25 \pm 3)$ & - \\
\hline Turbidez & 12 & $(147-261)$ & $(203 \pm 28)$ & $(3-49)$ & $(14 \pm 11)$ & 93,0 \\
\hline $\begin{array}{l}\text { Coliformes } \\
\text { termotolerantes }\end{array}$ & 12 & $\left(1 \times 10^{2}-16,3 \times 10^{6}\right)$ & $\left(9,21 \times 10^{6} \pm 4,5 \times 10^{6}\right)$ & $\left(0-6 \times 10^{4}\right)$ & $\left(6,5 \times 10^{3} \pm 17,1 \times 10^{3}\right)$ & 99,9 \\
\hline Coliformes totais & 12 & $\left(13 \times 10^{6}-72 \times 10^{6}\right)$ & $\left(33,75 \times 10^{6} \pm 14,9 \times 10^{6}\right)$ & $\left(10-9 \times 10^{4}\right)$ & $\left(34,5 \times 10^{3} \pm 21,9 \times 10^{3}\right)$ & 99,9 \\
\hline
\end{tabular}

Nota: $\mathrm{DBO}=$ demanda bioquímica de oxigênio $\left(\mathrm{mgO}_{2} \cdot \mathrm{L}^{-1}\right) ; \mathrm{DQO}=$ demanda química de oxigênio $\left(\mathrm{mgO}_{2} \cdot \mathrm{L}^{-1}\right) ; \mathrm{pH}=$ potencial hidrogeniônico; SD60 = sólidos sedimentáveis $(\mathrm{mg} \mathrm{L}-1) \mathrm{STT}=$ sólidos suspensos totais $\left(\mathrm{mg} . \mathrm{L}^{-1}\right) ; \mathrm{ST}=$ sólidos totais $\left(\mathrm{mg} . \mathrm{L}^{-1}\right)$; Temperatura $\left({ }^{\circ} \mathrm{C}\right)$; Turbidez (NTU); coliformes termotolerantes (UFC / $100 \mathrm{~mL}$ ); coliformes totais (UFC / $100 \mathrm{~mL}$ ); $\mathrm{n}$ = número amostral; DP = desvio padrão.

Todos estes parâmetros físicos, químicos e microbiológicos atenderam as condições e padrões estabelecidos na Resolução $n^{\circ} .430$, de 13 de maio de 2011, a qual dispõe sobre as condições e padrões de lançamento de efluentes, complementa e altera a Resolução n.357, de 17 de março de 2005, do Conselho Nacional do Meio Ambiente (CONAMA,2011).

\subsection{Pesquisa de cisto de Giardia spp. e oocistos de Cryptosporidium spp.}

Nas análises realizadas no período de fevereiro de 2015 a janeiro de 2016 (Tabelas 2 e 3) foram detectadas elevadas concentrações de cistos de Giardia spp. e oocistos de Cryptosporidium spp. 
No efluente bruto (P1) da ETE Garcia foram detectados cistos de Giardia spp. em $100 \%$ das amostras (12/12) em concentrações que variaram de 20.000 (min.) a 60.000 (máx.) e média de 26.666 cistos $\mathrm{L}^{-1}$, oocistos de Cryptosporidium spp. foram detectados em 16,7\% das amostras (2/12) em concentrações que variaram de 10.000 (min.) a 20.000 (máx.) e média de 2.500 oocistos $\mathrm{L}^{-1}$, indicando que formas infectantes de Giardia spp. e Cryptosporidium spp. circulam no ambiente dos bairros atendidos pela ETE e que parte da população humana e/ou animal pode estar infectada.

Greinert-Goulart (2013) também detectou cistos de Giardia spp. em 100\% amostras de esgoto bruto, em concentração média de 98.333 cistos L-1, e oocistos de Cryptosporidium spp. foram detectados em apenas um mês, dos 24 meses de análises, na pesquisa realizada na ETE Piçarrão, no município de Campinas, SP. O sistema de tratamento adotado na ETE Piçarrão é de Reator Anaeróbio de Fluxo Ascendente seguido de Lodos Ativados Convencional. A ETE Piçarrão, durante o estudo, contribuía para o tratamento de $23 \%$ do esgoto de Campinas, atendendo uma população de cerca de 200.000 habitantes.

Nasser et al. (2012) observaram após revisão de 30 estudos realizados em diferentes países que $76,6 \%$ das amostras analisadas de efluente bruto apresentaram positividade para Giardia spp., em concentrações que variaram de 0,23 a 100.000 cistos $\mathrm{L}^{-1}$. Esses autores concluíram que a elevada prevalência de cistos de Giardia em águas residuais em várias partes do mundo, pode refletir a taxa de infecção na população humana.

Já em relação ao Cryptosporidium spp., Nasser (2016) mediante a análise de 25 estudos conduzidos em 13 países, constatou a prevalência do protozoário entre $6 \%$ e 100\% das amostras de esgoto bruto analisadas com concentrações que variaram de 10 a 200 oocistos $\mathrm{L}^{-1}$.

A menor ocorrência de oocistos de Cryptosporidium em relação aos cistos de Giardia em amostras de efluentes brutos já foi observada em outros estudos (Cantusio Neto et al., 2006; Greinert-Goulart, 2013; Nasser et al., 2012; Nasser, 2016) e pode ser influenciada por diversos fatores, dentre os quais o principal é a prevalência da parasitose na população que utiliza os serviços de coleta e tratamento de esgoto doméstico.

Corroborando também com os resultados de Andrade et al. (2008) em uma pesquisa realizada no município de Blumenau, SC, em um Centro de Educação Infantil Público, no qual observaram a prevalência de Giardia duodenalis em 18,9\% das crianças analisadas e Cryptosporidium spp. em apenas 7,6\%. 
Tabela 2. Estimativa do número de cistos de Giardia spp. $\mathrm{L}^{-1}$ detectados nas diferentes etapas de tratamento da ETE Garcia, município de Blumenau, SC.

\begin{tabular}{|c|c|c|c|c|c|c|c|}
\hline \multirow[b]{2}{*}{ Meses } & \multirow[b]{2}{*}{ P1 } & \multirow[b]{2}{*}{$\mathbf{P 2}$} & \multicolumn{2}{|c|}{ Pontos de coleta } & \multirow[b]{2}{*}{ P5 } & \multirow[b]{2}{*}{ P6 } & \multirow[b]{2}{*}{ \% de Remoçãa } \\
\hline & & & $\mathbf{P 3}$ & $\mathbf{P 4}$ & & & \\
\hline Fev/15 & 20.000 & 60.000 & 150 & 20.000 & ND & 400.000 & 99,9 \\
\hline Mar/15 & 20.000 & 40.000 & 150 & ND & ND & 400.000 & 99,9 \\
\hline Abr/15 & 20.000 & 60.000 & ND & 20.000 & ND & 60.000 & 99,9 \\
\hline Mai/15 & 40.000 & 30.000 & 150 & 20.000 & ND & 60.000 & 99,9 \\
\hline Jun/15 & 60.000 & 40.000 & 100 & ND & ND & 100.000 & 99,9 \\
\hline $\mathrm{Jul} / 15$ & 20.000 & 40.000 & 150 & ND & ND & 100.000 & 99,9 \\
\hline Ago/15 & 20.000 & 60.000 & 350 & 10.000 & ND & 300.000 & 99,9 \\
\hline Set/15 & 20.000 & 40.000 & ND & 20.000 & ND & 300.000 & 99,9 \\
\hline Out/15 & 20.000 & 60.000 & 154 & ND & ND & 250.000 & 99,9 \\
\hline Nov/15 & 30.000 & 160.000 & 308 & 10.000 & ND & $\mathrm{NR}^{*}$ & 99,9 \\
\hline Dez/15 & 30.000 & 60.000 & 546 & 20.000 & ND & $\mathrm{NR}^{*}$ & 99,9 \\
\hline Jan/16 & 20.000 & 80.000 & ND & ND & ND & 900.000 & 99,9 \\
\hline Média & 26.666 & 60.833 & 171,42 & 10.000 & 0 & 329.167 & 99,9 \\
\hline DP & 11.785 & 32.777 & 154,32 & 9.128 & 0 & 260.175 & - \\
\hline $\mathrm{F}(\%)$ & 100,0 & 100,0 & 75,0 & 58,3 & 0,0 & 100,0 & - \\
\hline
\end{tabular}

Legenda: $\mathrm{ND}=$ não detectado; $\mathrm{NR}^{*}=$ não realizado; $\mathrm{P} 1$ = esgoto bruto; $\mathrm{P} 2=$ lodo UASB; $\mathrm{P} 3$ = efluente UASB; P4 = efluente MBBR; P5 = efluente tratado; P6 = lodo condicionado; $\mathrm{DP}=$ desvio padrão; $\mathrm{F}(\%)=$ frequência relativa.

Tabela 3. Estimativa do número de oocistos de Cryptosporidium spp. $\mathrm{L}^{-1}$ detectados nas diferentes etapas de tratamento da ETE Garcia, município de Blumenau, SC.

\begin{tabular}{lllllllc}
\hline & \multicolumn{7}{c}{ Pontos de coleta } \\
Meses & P1 & P2 & P3 & P4 & P5 & P6 & \% de Remoção \\
\hline Fev/15 & 20.000 & 60.000 & 50 & ND & ND & 200.000 & 99,9 \\
Mar/15 & ND & 40.000 & ND & ND & ND & ND & NA** \\
Abr/15 & ND & 60.000 & ND & ND & ND & ND & NA** \\
Mai/15 & ND & ND & ND & ND & ND & ND & NA** \\
Jun/15 & ND & ND & ND & ND & ND & ND & NA** \\
Jul/15 & ND & 40.000 & ND & ND & ND & 100.000 & NA** \\
Ago/15 & ND & ND & ND & ND & ND & 150.000 & NA** \\
Set/15 & ND & ND & ND & ND & ND & 300.000 & NA** \\
Out/15 & ND & ND & ND & ND & ND & ND & NA** \\
Nov/15 & ND & 80.000 & ND & ND & ND & NR** & NA** \\
Dez/15 & 10.000 & 40.000 & ND & ND & ND & NR** & 99,9 \\
Jan/16 & ND & ND & ND & ND & ND & 300.000 & NA** \\
\hline Média & 2.500 & 26.667 & 4,166 & 0 & 0 & 87.500 & 99,9 \\
DP & 5.951 & 28.674 & 13,81 & 0 & 0 & 115.695 & - \\
F $(\%)$ & 16,7 & 50,0 & 8,3 & 0,0 & 0,0 & 41,7 & - \\
\hline
\end{tabular}

Legenda: $\mathrm{ND}=$ não detectado; $\mathrm{NR}^{*}=$ não realizado; $\mathrm{NA}^{* *}=$ não avaliado $\mathrm{P} 1$ = esgoto bruto; $\mathrm{P} 2$ = lodo UASB; P3 = efluente UASB; P4 = efluente MBBR; P5 = efluente tratado; P6 = lodo condicionado; DP = desvio padrão; F $(\%)=$ frequência relativa. 
O ponto amostral (P2), referente ao lodo UASB (Upflow Anaerobic Sludge Blanket - reator anaeróbio de fluxo ascendente), apresentou cistos de Giardia spp. em 100,0\% das amostras analisadas em concentrações que variaram de 30.000 (min.) a 160.000 (máx.) e média de 60.833 cisto $\mathrm{L}^{-1}$, oocistos de Cryptosporidium spp. também estavam presentes em $50 \%$ das amostras em concentrações que variaram de 40.000 (min.) a 80.000 (máx.) e média de 26.667 oocistos $\mathrm{L}^{-1}$ evidenciando o acúmulo dos protozoários nos sistemas de retenção de lodo da estação.

Greinert-Goulart (2013) também detectou cistos de Giardia spp. em 100,0\% amostras de lodo RAFA (reator anaeróbio de fluxo ascendente) em concentração média de 281.167 cistos $\mathrm{L}^{-1}$. Já os oocistos de Cryptosporidium spp. foram detectados em apenas um mês, em concentração de 80.000 oocistos $\mathrm{L}^{-1}$.

No efluente UASB (P3) foram detectados cistos de Giardia spp. em $75 \%$ das amostras analisadas em concentrações que variaram de 100 (min.) a 545,45 (máx.) e média de 171,42 cisto $\mathrm{L}^{-1}$, oocistos de Cryptosporidium spp. foram detectados em apenas $8,3 \%$ das amostras (1/12) com concentração de 50 oocistos $\mathrm{L}^{-1}$ evidenciando a eficiência de remoção destes protozoários no reator UASB.

Greinert-Goulart (2013) detectou cistos de Giardia spp. em 100,0\% das amostras de efluente RAFA em concentração média de 193 cistos $\mathrm{L}^{-1}$. Já os oocistos de Cryptosporidium spp. em amostras de efluente RAFA foram detectados em apenas um mês, dos 24 meses de análises, em concentração de 100 oocistos $\mathrm{L}^{-1}$.

No efluente MBBR (P4) da ETE Garcia foram detectados cistos de Giardia spp. em 58,33\% dos pontos analisados, em concentrações que variaram de 10.000 (min.) a 20.000 (máx.) e média de 10.000 cistos $\mathrm{L}^{-1}$, oocistos de Cryptosporidium spp., não foram detectados neste ponto demonstrando a eficiência de remoção destes protozoários pelo sistema MBBR.

Greinert-Goulart (2013) detectou cistos de Giardia spp. em 100,0\% das amostras de lodo do tanque de aeração em concentração média de 123.667 cistos $\mathrm{L}^{-1}$. Oocistos de Cryptosporidium spp. não foram detectados neste reator corroborando com os resultados obtidos nesta pesquisa.

Nos sistemas de tratamento aeróbios, como MBBR e lodos ativados, estudos indicam que pode ocorrer a remoção destes parasitos por predação exercida por componentes da microfauna presente no tanque de aeração (Stott et al., 2001; 2003; Fayer et al., 2000). Na ETE Piçarrão, no município de Campinas, Siqueira-Castro et al. (2016) observaram que os ciliados de vida livre Euplotes aediculatus e Sterkiella cavicola componentes na microfauna do sistema de lodo ativado demonstraram potencial predatório sobre oocistos de Cryptosporidium spp. e cistos de Giardia spp. Estes resultados indicam que a predação por protozoários de vida livre pode ser um importante fator na remoção de cistos e oocistos de efluentes de esgoto doméstico.

No efluente tratado (P5) não foram detectados cistos de Giardia spp. e oocistos de Cryptosporidium spp., demonstrando uma remoção de 99,9\% destes protozoários pelo sistema combinado da ETE Garcia.

Resultados semelhantes também foram observados por Cantusio Neto et al. (2006) na ETE Samambaia, Greinert-Goulart (2013) na ETE Piçarrão ambos no município de Campinas e por Cheng et al. (2009) na avaliação de quatro ETE irlandesas que utilizavam sistema de lodos ativados ou biofiltro percolador para o tratamento secundário de efluentes domésticos.

O maior tempo de detenção hidráulica do efluente dentro dos reatores anaeróbios e aeróbios pode contribuir para a maior eficiência na remoção dos cistos e oocistos (Nasser et al., 2012; Nasser 2016). O sistema combinado de tratamento anaeróbio + aeróbio da ETE Garcia foi projetado para tratar $88 \mathrm{~L} . \mathrm{s}^{-1}$, porém, no período da pesquisa operou com apenas $30 \mathrm{~L} . \mathrm{s}^{-1}$ aumentando deste modo o tempo de detenção hidráulica do efluente dentro dos reatores da ETE Garcia $(\mathrm{TDH}=34 \mathrm{~h}$ ), o que pode ter contribuído para as altas taxas de remoção observadas. 
Ressalta-se que estes sistemas de tratamento de esgoto doméstico em nível secundário não foram projetados para a total remoção destes protozoários. Para cada sistema é necessário realizar uma avaliação cautelosa da qualidade do efluente final para o conhecimento das taxas de remoção e a necessidade de inclusão de um tratamento terciário adicional para evitar a contaminação ambiental devido a presença de protozoários patogênicos mesmo em efluentes tratados como já demostrado em diversos estudos (Nasser et al. 2012; Hofstra et al., 2013; Taran-Benshoshan et al., 2015; Nasser, 2016).

Neste estudo o sistema apresentou altas taxas de remoção o que demostra a evidente contribuição do tratamento de esgoto na redução da exposição das populações humana e animal ao risco de contaminação por patógenos em função do lançamento de esgoto não tratado em mananciais superficiais.

Nas amostras de lodo condicionado (P6) (subproduto final da estação) foram detectados cistos de Giardia spp. em todas as amostras analisadas, com concentrações que variaram de 60.000 (min.) a 900.000 (máx.), e média de 329.167 cistos $\mathrm{L}^{-1}$. Oocistos de Cryptosporidium spp. também estavam presentes em $41,7 \%$ das amostras, com concentrações que variaram de 100.000 (min.) a 300.000 (máx.), e média de 87.500 oocistos $\mathrm{L}^{-1}$.

Em outros estudos que avaliaram lodo condicionado (Bonatti et al., 2007; Cheng et al., 2009; Greinert-Goulart, 2013) foram observadas concentrações menores de cistos e oocistos. Possivelmente, pela constituição física do lodo condicionado da ETE Garcia, o qual é resultado da reunião de vários descartes de lodo recirculado do sistema UASB, de lodo recirculado do sistema MBBR e materiais particulados do decantador secundário, submetidos a processos de centrifugação com adição de polímeros. Este processo pode ter contribuído para o aumento da taxa de sólidos totais e o acúmulo de cistos e oocistos neste sistema de descarte.

De acordo com a Resolução CONAMA N $375 / 2006$, retificada pela Resolução CONAMA $\mathrm{N}^{\mathrm{o}} 380 / 2002$, o lodo de esgoto corresponde a uma fonte potencial de riscos à saúde pública e ao ambiente, potencializando a proliferação de vetores de moléstias e organismos nocivos, sendo necessária a adoção de medidas para dispor esse material de forma a não causar nenhum dano ao meio ambiente e a sociedade (CONAMA, 2006). Deste modo a NBR 10.004 (ABNT, 2004) alerta para os riscos com a manipulação, transporte e disposição final deste resíduo classificado como classe II - não inertes.

O sistema combinado empregado na ETE Garcia demostrou altas taxas de remoção de cistos e oocistos a partir do esgoto bruto e o acúmulo destes no lodo condicionado. Estes resultados ressaltam a necessidade de ampliação dos serviços de tratamento de esgoto no município de Blumenau, bem como em todo o país, para redução da contaminação ambiental de mananciais superficiais e, consequentemente contribuindo para melhoria na saúde pública reduzindo o risco de infecção da população humana e animal.

\subsection{Análise de correlação entre os resultados quantitativos da pesquisa de oocistos de Cryptosporidium spp. e cistos de Giardia spp. e as concentrações dos parâmetros físicos, químicos e microbiológicos}

Após realização das análises do coeficiente de correlação de Spearman foi observada correlação negativa significativa entre a concentração de oocistos de Cryptosporidium e a concentração de coliformes termotolerantes no efluente bruto $(r=-0.69 ; P=0,012 ; n=12)$. Outros autores demonstram correlações significativas, porém positivas entre a concentração de oocistos de Cryptosporidium spp. e concentração de coliformes termotolerantes, coliformes totais e turbidez (Xiao et al., 2013). As demais correlações entre a concentração de oocistos de Cryptosporidium spp. e os demais parâmetros avaliados foram consideradas fracas ou nulas.

Observou-se correlação negativa significativa ( $r=-0,57 ; P=0,048 n=12$ ) entre a concentração de cistos de Giardia e a turbidez do efluente bruto, as demais correlações entre a concentração de cistos de Giardia spp. e os demais parâmetros avaliados foram consideradas 
fracas ou nulas. Correlações negativas entre a densidade de cistos, oocistos e turbidez também foram observadas por Kumar et al. (2016), indicando que a recuperação destes protozoários pode diminuir à medida que a turbidez das amostras aumenta muito, sendo necessário a melhoria das técnicas de detecção para amostras com elevada turbidez.

Porém, no estudo realizado por Greinert-Goulart (2013) foram observadas correlações positivas entre a concentração de protozoários e a turbidez do efluente do UASB. Segundo Cantusio Neto et al. (2010), características da amostra de água, tais como, sílica, presença de ácidos húmicos, bactérias, algas, metais, matéria orgânica em decomposição, bem como variações do $\mathrm{pH}$ e da turbidez, são fatores críticos que podem afetar negativamente a sensibilidade das diversas metodologias para detecção de cistos e oocistos.

\subsection{Análise de variação sazonal dos resultados quantitativos da pesquisa de cistos de Giardia spp. e oocistos de Cryptosporidium spp.}

Nas análises de variação sazonal da concentração de cistos de Giardia spp. em amostras de efluente bruto não foram encontradas diferenças significativas $(p=0,13$; $F=2,46 ; F$ crítico $=4,06 ; n=12$ ), do mesmo modo que não foi observado a variação sazonal na concentração de oocistos de Cryptosporidium spp. em amostras de efluente bruto ( $p=0,56$; $F=0,73 ; F$ crítico $=4,06 ; n=12)$. Em relação ao efluente tratado não foi realizada análises de correlação em razão de não ter sido detectada a presença de cistos de Giardia spp. e oocistos de Cryptosporidium spp.

Diversos estudos apresentaram variações na concentração de cistos de Giardia spp. e oocistos de Cryptosporidium spp. em amostras de efluente bruto e efluente tratado em diversas estações do ano, e afirmam que a distribuição sazonal da concentração de cistos de Giardia spp. e oocistos de Cryptosporidium spp. no esgoto bruto varia de acordo com taxa de infecção da população humana e com as características climáticas do local de estudo (Castro-Hermida et al., 2008; Cheng et al., 2009; Nasser et al., 2012; Nasser, 2016).

\section{CONCLUSÃO E RECOMENDAÇÕES}

Nesse estudo, demonstrou-se a presença dos protozoários patogênicos Giardia spp. e Cryptosporidium spp. no esgoto bruto, durante os processos de tratamento e nos subprodutos finais da ETE Garcia, deste modo qualquer falha operacional na ETE pode ocasionar a contaminação do principal manancial da região (Rio Itajaí-Açu), sendo este destinado ao abastecimento público de vários municípios a jusante da Estação (i.e., Gaspar, Ilhota, Itajaí).

Portanto, faz-se necessário, o monitoramento destes efluentes, a disposição final adequada do lodo biológico e a padronização de técnicas apropriadas para a detecção desses protozoários em amostras ambientais.

Apesar da grande relevância que estes protozoários de veiculação hídrica apresentam à saúde pública, aliada a resistência de suas formas infectantes aos processos físicos, químicos e microbiológicos comumente utilizados pelas estações de tratamento de água e esgoto sanitário, estes não são levados em consideração no monitoramento da qualidade dos efluentes gerados pelas estações de tratamento de esgoto sanitário.

Assim, diversas estações de tratamento de esgoto podem estar contribuindo para a contaminação dos mananciais destinados ao abastecimento público, expondo a população que utiliza os mesmos a riscos de infecção por protozoários patogênicos de veiculação hídrica.

Deste modo, a compreensão da função particular de cada sistema de tratamento de esgoto torna-se necessária, no sentido de identificar que etapas do processo contribuirão para a remoção destes protozoários, e otimização destes processos para a total remoção/inativação das formas infectantes destes protozoários, a fim de minimizar os impactos adversos provenientes do lançamento de efluentes gerados pelas ETE no meio ambiente e na Saúde Pública. 
Embora não tenha sido detectada a presença destes protozoários em amostras de efluente tratado, a utilização de ferramentas moleculares como o qPCR, para a identificação das assembleias/espécies de Giardia e Cryptosporidium e a viabilidade/infectividade de cistos e oocistos presentes no efluente bruto, serão importantes ferramentas a serem utilizadas futuramente para avaliar corretamente os riscos de infecção a população humana.

\section{AGRADECIMENTOS}

À Coordenação de Aperfeiçoamento de Pessoal em Nível Superior (CAPES) e à Fundação de Amparo à Pesquisa e Inovação do Estado de Santa Catarina (FAPESC) pela concessão da bolsa de mestrado ao primeiro autor. À Fundação Universidade Regional de Blumenau (FURB) por meio do Programa de Pós-Graduação em Engenharia Ambiental pela orientação e uso de laboratórios e equipamentos. À empresa Odebrecht Ambiental LTDA pela autorização e informações pertinentes ao sistema que tratamento de esgoto sanitário do município de Blumenau.

\section{REFERÊNCIAS}

AMERICAN PUBLIC HEALTH ASSOCIATION - APHA. Standard methods for the examination of water and wastewater. 22nd ed. Washington, DC, 2012.

ANDRADE, J.; RODE, G.; SILVA FILHO, H. H.; GREINERT-GOULART, J. A. Parasitoses intestinais em um centro de educação infantil público do município de Blumenau (SC), Brasil, com ênfase em Cryptosporidium spp. e outros protozoários. Revista de patologia tropical, v. 37, n. 4, p. 332-340, 2008. https://doi.org/10.5216/rpt.v37i4.5665

ASSOCIAÇÃO BRASILEIRA DE NORMAS TÉCNICAS - ABNT. NBR 10004: Resíduos sólidos - Classificação. Rio de Janeiro, 2004.

BALDURSSON, S.; KARANIS, P. Waterborne transmission of protozoan parasites: review of worldwide outbreaks - an update 2004-2010. Water Research, v. 45, p. 6603-6614, 2011. http://dx.doi.org/10.1016/j.watres.2011.10.013

BLUMENAU. Blumenau entre as $\mathbf{1 0 0}$ cidades com tratamento de esgoto no país. Blumenau, 2016. Disponível em: http://www.blumenau.sc.gov.br/secretarias/samae/samae/blumenau-entre-as-100cidades-com-tratamento-de-esgoto-no-pais87. Acesso em: 25 jul. 2016.

BONATTI, T. R.; FRANCO, R. M. B.; CANTUSIO NETO, R. Comparison of two methodologies for detection of Giardia spp. cysts and Cryptosporidium spp. oocysts in activated sludge from a sewage treatment plant in the city of Campinas, São Paulo, Brazil. Journal of Water and Health, v. 05, p. 1-7, 2007. http://dx.doi.org/10.2166/wh.2007.006

CANTUSIO NETO, R.; FRANCO, R. M. B. Ocorrência de oocistos de Cryptosporidium spp. e cistos de Giardia spp. em diferentes pontos do processo de tratamento de água, em Campinas, São Paulo, Brasil. Higiene Alimentar, v. 18, n. 118, p. 52-59, 2004.

CANTUSIO NETO, R.; SANTOS, L. U.; FRANCO, R. M. B. Evaluation of activated sludge treatment and the efficiency of the disinfection of Giardia species cysts and Cryptosporidium oocysts by UV at a sludge treatment plant in Campinas, southeast Brazil. Water Science and Technology, v. 54, n. 3, p. 89-94, 2006. http://dx.doi.org/10.2166/wst.2006.453 
CANTUSIO NETO, R.; SANTOS, L. U.; SATO, M. I. Z.; FRANCO R. M. B. Cryptosporidium spp. and Giardia spp. in surface water supply of Campinas, Southeast Brazil. Water Science \& Technology, v. 62, n. 1, p. 217-222, 2010. http://dx.doi.org/10.2166/wst.2010.312

CASTRO-HERMIDA, J. A.; GARCÍA-PRESEDO, I.; ALMEIDA, A.; GONZÁLEZWARLETA, M.; CORREIA, C. J. M.; MEZO, M. Contribution of treated wastewater to the contamination of recreational river areas with Cryptosporidium spp. and Giardia duodenalis. Water Research, v. 42, n. 13, p. 3528-38, 2008. http://dx.doi.org/10.1016/j.watres.2008.05.001

CHAPPELL, C. L.; OKHUYSEN, P. C.; LANGER-CURRY, R. C.; LUPO, P. J.; WIDMER, G.; TZIPORI, S. Cryptosporidium muris: Infectivity and Illness in Healthy Adult Volunteers. American Journal of Tropical Medicine and Hygiene, v. 92, n. 1, p. 50 55, 2015. http://dx.doi.org/10.4269/ajtmh.14-0525

CHENG, H. A.; LUCY, F. E.; GRACZYK, T. K.; BROADERS, M. A.; TAMANG, L.; CONNOLLY, M. Fate of Cryptosporidium parvum and Cryptosporidium hominis oocysts and Giardia duodenalis cysts during secondary wastewater treatments. Parasitology Research, v. 105, p. 689-696, 2009. http://dx.doi.org/10.1007/s00436009-1440-y

CONSELHO NACIONAL DO MEIO AMBIENTE - CONAMA. Resolução 375, de 29 de agosto de 2006. Define critérios e procedimentos, para o uso agrícola de lodos de esgoto gerados em estações de tratamento de esgoto sanitário e seus produtos derivados, e dá outras providências. Diário Oficial [da] União, Brasília, 30 ago. 2006.

CONSELHO NACIONAL DO MEIO AMBIENTE - CONAMA. Resolução 430, de 13 de maio de 2011. Dispõe sobre as condições e padrões de lançamento de efluentes, complementa e altera a Resolução no 357, de 17 de março de 2005, do Conselho Nacional do Meio Ambiente-CONAMA. Diário Oficial [da] União, Brasília, n. 92, 16 maio 2011, p. 89.

FAYER, R.; TROUT, J. M.; WALSH, E.; COLE, R. Rotifers ingest oocysts of Cryptosporidium parvum. Journal of Eukaryotic Microbiology, v. 47, n. 2, p. 161-163, 2000. http://dx.doi.org/10.1111/j.1550-7408.2000.tb00026.x

FOOD AND AGRICULTURE ORGANIZATION; WORLD HEALTH ORGANIZATION. Multicriteria-based ranking for risk management of food-borne parasites. Rome, 2014. 324 p. (Microbiological Risk Assessment Series)

FRANCO, R. M. B.; ROCHA-EBERHARDT, R.; CANTUSIO NETO, R. Occurence of Cryptosporidium oocysts and Giardia cysts in raw water from the Atibaia river, Campinas, Brazil. Revista do Instituto de Medicina Tropical de São Paulo, v. 43, n. 2, p. 109-111, 2001. http://dx.doi.org/10.1590/S0036-46652001000200011

FREGONESI, B. M.; SAMPAIO, C. F.; RAGAZZI, M. F.; TONANI, K. A. A.; SEGURAMUÑOZ, S. I. Cryptosporidium e Giardia: desafios em águas de abastecimento público. O Mundo da Saúde, v. 36, n. 4, p. 602-609, 2012.

GREINERT-GOULART, J. A. Estudo da remoção de cistos de Giardia spp. e oocistos de Cryptosporidium spp. e caracterização da microfauna em estações de tratamento de esgoto: instrumentos de avaliação e controle do processo. 2013. 196f. Tese (Programa de Pós-Graduação Instituto de Biologia) - Universidade Regional de Campinas, Campinas, 2013. 
GROTT, S. C.; HARTMANN, B.; SILVA FILHO, H. H.; FRANCO, R. M. B.; GREINERTGOULART, J. A. Detecção de cistos de Giardia spp. e oocistos de Cryptosporidium spp. na água bruta das estações de tratamento no município de Blumenau, SC, Brasil. Revista Ambiente \& Água, v. 11, n. 3, 2016. http://dx.doi.org/10.4136/ambi-agua.1853

HOFSTRA, N.; BOUWMAN, A. F.; BEUSEN, A. H. W.; MEDEMA, G. J. Exploring global Cryptosporidium emissions to surface water. Science of the Total Environment, v. 442, p. 10-19, 2013. http://dx.doi.org/10.1016/j.scitotenv.2012.10.013

KARANIS, P.; KOURENTI, C.; SMITH, H. Waterborne transmission of protozoan parasites: A worldwide review of outbreaks and lessons learnt. Journal of Water and Health, v. 5, n. 1, p. 1-38, 2007. http://dx.doi.org/10.2166/wh.2006.002

KUMAR, T.; MAJID, M. A. A.; ONICHANDRAN, S.; JATURAS, N.; ANDIAPPAN, H.; SALIBAY, C. C. et al. Presence of Cryptosporidium parvum and Giardia lamblia in water samples from Southeast Asia: towards an integrated water detection system. Infectious Diseases of Poverty, V. 5, N. 3, 2016. https://doi.org/10.1186/s40249-0160095-Z

NASSER, A. M.; VAIZEL-OHAYON, D.; AHARONI, A.; REVHUN, M. Prevalence and fate of Giardia cysts in wastewater treatment plants. Journal of Applied Microbiology, v. 113, p. 477-484, 2012. http://dx.doi.org/10.1111/j.1365-2672.2012.05335.x

NASSER, A. M. Removal of Cryptosporidium by wastewater treatment processes: a review. Journal of Water and Health, v. 14, n. 1, p. 1-13, 2016. http://dx.doi.org/10.2166/wh.2015.131

ODEBRECHT AMBIENTAL. Website. Disponível em: http://www.odebrechtambiental.com. Acesso em: 22 maio 2016.

PUTIGNANI, L.; MENICHELLA, D. Global distribution, public health and clinical impact of the protozoan pathogen Cryptosporidium. Interdisciplinary Perspectives on Infectious Diseases, v. 753, n. 512, 2010. http://dx.doi.org/10.1155/2010/753512

ROBERTSON, L. J.; PATON, C. A.; CAMPBELL, A. T.; SMITH, P. G.; JACKSON, M. H.; GILMOUR, R. A. et al. Giardia cysts and Cryptosporidium oocysts at sewage treatment works in Scotland, UK. Water Research, v. 34, n. 8, p. 2310-2322, 2000.

SAVIOLI, L.; SMITH, H.; THOMPSON, A. Giardia and Cryptosporidium join the "Neglected Diseases Initiative". Trends in Parasitology, v. 22, n. 5, p. 203-208, 2006. http://dx.doi.org/10.1016/j.pt.2006.02.015

SIQUEIRA-CASTRO, I. C. V.; GREINERT-GOULART, J. A.; BONATTI, T. R.; YAMASHIRO, S.; FRANCO, R. M. B. First report of predation of Giardia sp. cysts by ciliated protozoa and confirmation of predation of Cryptosporidium spp. oocysts by ciliate species. Environmental Science Pollution Research, 2016. http://dx.doi.org/10.1007/s11356-016-6689-y

SISTEMA NACIONAL DE INFORMAÇÕES SOBRE SANEAMENTO. Diagnóstico dos serviços de água e esgotos - 2012. Brasília, Secretaria Nacional de Saneamento Ambiental, 2014.

STOTT, R.; MAY, E.; MATSUSHITA, E.; WARREN, A. Protozoan predation as a mechanism for the removal of Cryptosporidium oocysts from wastewaters in constructed wetlands. Water Science and Technology, v. 44, n. 11-12, p. 191-198, 2001. 
STOTT, R.; MAY, E.; RAMIREZ, E.; WARREN, A. Predation of Cryptosporidium oocysts by protozoa and rotifers: implications for water quality and public health. Water Science and Technology, v. 47, n. 3, p. 77-83, 2003.

TARAN-BENSHOSHAN, M.; OFER, N.; DALIT, V. O.; AHARONI, A.; REVHUN, M.; NITZAN, Y. et al. Cryptosporidium and Giardia removal by secondary and tertiary wastewater treatment. Journal of environmental science and health. Part A, Toxic/hazardous substances \& environmental engineering, v. 50, n. 12, p. 1265-1273, 2015. http://dx.doi.org/10.1080/10934529.2015.1055152

TRATA BRASIL. Saneamento, nova realidade: o Brasil está muito longe do ideal. 2013. Disponível em: http://www.tratabrasil.org.br/novo_site/?id=5760. Acesso em: 19 jul. 2016.

UNITED NATIONS CHILDREN'S FUND - UNICEF, WORLD HEALTH ORGANIZATION - WHO. Diarrhoea: why children are still dying and what can be done. New York: Unicef; Geneva: WHO, 2009. 58 p.

VOLOTÃO, A.; SOUZA JUNIOR, J. C.; GRASSINI, C.; PERALTA, J. M.; FERNANDES, O. Genotyping of Giardia duodenalis from Southern brown howler monkeys (Alouatta clamitans) from Brazil. Veterinary Parasitology, v. 158, p. 133-137, 2008. http://dx.doi.org/10.1016/j.vetpar.2008.07.003

WORLD HEALTH ORGANIZATION - WHO. Guidelines for drinking-water quality. 4th ed. Geneva, 2011.

WORLD HEALTH ORGANIZATION - WHO. Investing in water and sanitation: increasing access, reducing inequalities. UN-Water Global Analysis and Assessment of Sanitation and Drinking-Water GLAAS 2014 Report. New York, 2014. p. 108.

XIAO, G.; QIU, Z.; QI, J.; CHEN, J.; LIU, F.; LIU, W. et al. Occurrence and potential health risk of Cryptosporidium and Giardia in the Three Gorges Reservoir, China. Water research, n. 47, p. 2431-2445, 2013. http://dx.doi.org/10.1016/j.watres.2013.02.019 